\title{
Reliability of COP Measures in Healthy and Low Back Pain Subjects During a Dynamic Task
}

\author{
Mokhtarinia HR ${ }^{1 *}$, Kahrizi S², Sanjari MA $^{3}$ and Parnianpour M4 \\ ${ }^{1}$ Department of Ergonomics, University of Social Welfare and Rehabilitation Sciences, \\ Iran \\ 2Department of Physical Therapy, School of Medical Sciences, Tarbiat Modares \\ University, Iran
}

${ }^{3}$ Department of Rehabilitation Basic Sciences, Tehran University

\section{Research Article \\ Volume 1 Issue 3}

Received Date: December 09, 2017

Published Date: December 26, 2017

DOI: $10.23880 /$ eoij-16000129

of Medical Sciences, Iran ${ }^{4}$ Department of Mechanical Engineering, Sharif University of Technology, Iran

*Corresponding author: Hamidreza mokhtarinia, phd, Department of Ergonomics, University of Social Welfare and Rehabilitation Sciences, Tehran, Iran, Tel: 9821 22180119; E-mail: Hrmokhtarinia@yahoo.com

\section{Abstract}

Study design. A test-retest design.

Objectives: To study the reliability of the postural stability measures during whole body dynamic conditions in healthy and low back pain (LBP) subjects.

Summary of Background Data: Postural control is an essential prerequisite for dynamic motions such as lifting, lowering, and repetitive trunk bending tasks. Test retest reliability of center of pressure (COP) measures is well documented in static conditions but such results may not be applicable to dynamic conditions. To the authors knowledge this has not been investigated in dynamic conditions.

Methods: Twenty four subjects (12 healthy and 12 LBP) performed repeated trunk bending in two different conditions of high and low speed in sagittal plane. All measurements were repeated and recorded with an interval of 7-10 days later.

COP data were used to calculate standard deviation of amplitude, standard deviation of velocity in anterior-posterior and medial-lateral directions, mean total velocity and path length. Relative reliability was assessed using intra class correlation coefficient (ICC) and absolute reliability using standard error of measurement (SEM), minimum detectable change (MMDC) and coefficient of variation (CV).

Results: Results in both groups revealed that mean total velocity and standard deviation of amplitude in anteriorposterior direction have an acceptable good to an excellent reliability with ICC range of 0.61-0.89. In healthy group the rest of others parameters also demonstrated an acceptable good to excellent reliability (range 0.6-0.97). In LBP subjects, in addition to standard deviation of amplitude in anterior posterior direction and mean total velocity, standard deviation of velocity in A-P in high pace condition also showed good reliability (ICC=0.69). 


\section{Ergonomics International Journal}

Conclusion: Mean total velocity and standard deviation of amplitude in anterior-posterior direction are suggested as a good reliable parameter to use for evaluation postural stability in dynamic situations such as lifting and complex trunk movement.

Keywords: Postural Stability; Whole body dynamic; Center of pressure; Reliability

Abbreviations: LBP: Low Back Pain; ICC: Intra Class Correlation Coefficient; SEM: Standard Error Of Measurement; MMDC: Minimal Metrically Detectable Change; CV: Coefficient of Variation; COP: Center Of Pressure; SD: Standard Deviation; AP: Anteroposterior; ML: Mediolateral.

\section{Introduction}

Balance or postural control is a health index of musculoskeletal system [1]. Balance skills during activity of daily life are needed to prevent one to fall and thus preventing their possible injuries [2]. Also balance control is an essential prerequisite for other dynamic motions such as lifting, lowering, twisting and repetitive trunk bending tasks [2-4]. Previous researches on the assessment of neuromuscular control of postural stability have concentrated on the static tasks [5,6]; while the significant role of dynamic activities in creating low back injuries are recognized, hence it seems that the postural assessment in dynamic situation can give more information about LBP $[6,7]$.

In contrast to static task, during dynamic trunk movement, postural control performance [8], joint movement patterns [9] and spinal stability $[6,10]$ can be affected by factors such as task asymmetry, speed of motion and existence of external load [11]. Force plate measurements (center of pressure position and displacement) are mostly used in static and quiet standing posture and their test retest reliability is well documented $[6,8,12]$, but such results may not be applicable to dynamic conditions $[5,6]$. Hence we have developed this study in response to identified void in the litectures. The main objective of this study was to assess test-retest reliability of various COP parameters in two different dynamic conditions of high and low speed trunk bending in healthy and LBP subjects.

\section{Materials and Methods}

\section{Participants}

Twelve male subjects with chronic nonspecific low back pain (NLBP), mean age $=30.5+6.48$ years, and 12 healthy male controls, mean age $=26.9+5.28$ years, were recruited from local physical therapy clinics and University of Social Welfare and Rehabilitation Sciences (USWR) staff. All participants were male because of the sex effect on cop measures. Inclusion criteria were: (1) ability to complete repetitive trunk bending movement, (2) ability to follow instructions, (3) no visual or hearing impairment, (4) no neuro-muscular or orthopedic dysfunction and/or taking any medications which renders the subject's balance[11,13]. LBP group have had a low back pain for at least 3 months and they were nearly pain free in the test condition because of high demanding movement tasks included the experimental protocol,(i.e. pain score lower than 2 on Visual Analogue Scale [14]. Healthy male participants were matched by age, height, weight, body mass index and physical fitness (mean weight $71.2 \pm 6.1 \mathrm{~kg}$, mean height $179 \pm 7 \mathrm{~cm}$, mean body mass index (BMI) $22.3 \pm 2.4 \mathrm{~kg} / \mathrm{m}^{2}$ ) to LBP participants (mean weight $70.25 \pm 12.1 \mathrm{~kg}$, mean height $173 \pm 8 \mathrm{~cm}$, mean BMI $23.4 \pm 3.8 \mathrm{~kg} / \mathrm{m}^{2)}$ ). All subjects read and signed an informed consent approved by the Tarbiat Modares University's Ethics Committee.

\section{Experimental Procedures}

COP data were acquired from a force plate (model Z812A, Kistler Instruments AG, Switzerland) which was located in the Ergonomics Lab of USWR by the same physical therapist (HR. M) in two sessions with one week interval. Subjects stood on the force plate with feet separated by shoulder width. All subjects were wanted to execute repeated trunk flexion-extension movements consecutively in the sagittal plane $[13,15]$. They were asked to touch a target with their hands located in the sagittal, $50 \mathrm{~cm}$ anterior to the knee in upright position, while looking at another target placed at shoulder height in the sagittal midline during standing. Specifically, the participants were asked to touch the lower target with extended arms, followed by looking at the upper target with enough extended trunk while their arms were positioned alongside their bodies, and to do this motion repeatedly throughout the duration of each trial. No motor constraints were imposed on the lower extremity joints since movement constraints affect motor control strategies $[13,15]$. In order to avoid excessive range of motion, subjects were asked not to move their knees or 
feet. Verbal feedback was given if instructions were violated (Figure1).
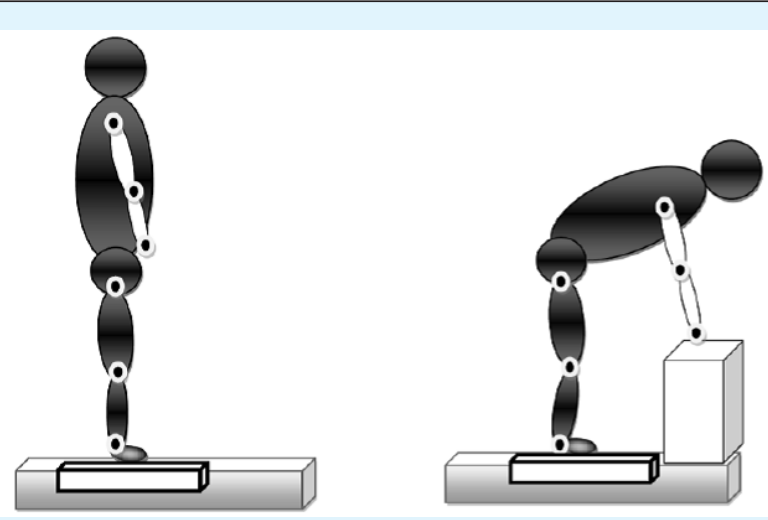

Figure1: Task condition from starting to bend position

The repetitive movements at two different speeds: 20 cycles/min and $40 \mathrm{cycles} / \mathrm{min}$ were included. Subjects were asked to reach targets synchronously with a metronome in order to establish the movement pace of 20 and 40 cycles/min similar to Granata, et al. experiment
[6]. Based on this movement pace, we wanted the subject having 30 cycles per each trial, so the slow and fast pace trials lasted 90 and 45 second in duration, respectively [16]. The subjects were allowed several practice trials prior to the main trial to get familiar with the task pace and order. In order to prevent fatigue effects, at least a 3 min rest period between each trial was allowed.

\section{Data Analysis}

The force plate output was filtered with a low pass Butterworth filter (cut-off frequency of $10 \mathrm{~Hz}$ ) and later the following parameters such as standard deviation (SD) of amplitude in anterior posterior (AP) and medial lateral (ML) directions (SDx and SDy), SD velocity in AP and ML directions (SDVx and SDVy) and mean total velocity (Vm) were determined. The formula used to calculate each parameter is presented in Table 1. To avoid transient's effects, the first five seconds of each recording was omitted. The remaining time series (45 seconds for high speed and 90 seconds for low speed conditions) were used for the computation of measures and subsequent statistical analysis to determine reliability measures.

\begin{tabular}{|c|c|}
\hline Parameter & Formula \\
\hline SD of amplitude $(\mathrm{cm})$ AP & $\sigma_{x}=\sqrt{\frac{\sum\left(x_{i}-\bar{x}\right)^{2}}{N-1}}$ \\
\hline ML & $\sigma_{y}=\sqrt{\frac{\sum\left(y_{i}-\bar{y}\right)^{2}}{N-1}}$ \\
\hline SD of velocity $(\mathrm{cm} / \mathrm{s}) \mathrm{AP}$ & $\sigma_{v_{x}}=\sqrt{\frac{\sum\left(v_{x_{i}}-\bar{v}\right)^{2}}{N-1}, \text { Where }} v_{x_{i}}=\frac{x_{i+1}-x_{i}}{t_{i+1}-t_{i}}$ \\
\hline ML & $\sigma_{v_{y}}=\sqrt{\frac{\sum\left(v_{y_{i}}-\bar{v}\right)^{2}}{N-1}}$, Where $v_{y_{i}}=\frac{y_{i+1}-y_{i}}{t_{i+1}-t_{i}}$ \\
\hline Mean total velocity $(\mathrm{cm} / \mathrm{s})$ & $\bar{v}=\frac{1}{T} \sum_{1}^{T} \sqrt{\left(x_{t+1}-x_{t}\right)^{2}+\left(y_{t+1}-y_{t}\right)^{2}}$ \\
\hline Path length (cm) & $P L=\sum_{i=1}^{T} \sqrt{\left(x_{i+1}-x_{i}\right)^{2}+\left(y_{i+1}-y_{i}\right)^{2}}$ \\
\hline
\end{tabular}

Table1: Formulae used to calculate COP parameters 


\section{Ergonomics International Journal}

\section{Statistical Analysis}

Reliability of each COP-based measure was assessed with interclass correlation coefficient (ICC) and standard error of measurement (SEM) [17]. We used two way random model of ICC to assess relative reliability, also known as $I C C_{2,1}$ [17]. ICC is determined as between subject variance versus total variance and was chosen to assess relative reliability $[18,19]$. ICC ranges were interpreted using the following criteria: $0.00-0.39$ poor, 0.40-0.59 fair, 0.60-0.74 good and 0.75-1.00 excellent [20]. The level of statistical significance set at 0.05 levels for all statistical data.

SEM and 95\% confidence intervals (CI) were also calculated for all dependent variables. SEM provides an estimate of the precision of measurement [17] and calculated as the square root of the mean square error term derived from the analysis of variance table [19]. A high SEM implies non-reproducibility of tested values.
Minimal metrically detectable change (MMDC) interpreted as the magnitude of change below which there is more than a $95 \%$ chance that no real change has occurred and defined as $95 \% \mathrm{CI}$ of SEM of COP measure $\left[\mathrm{MDC}_{90}=\mathrm{SEM} * 1.96 \sqrt{2}\right][21,22]$. Also, the coefficient of variation (CV) was determined for comparison of absolute reliability between different COP parameters (SD/Mean $\times 100$ ). It was achieved by calculating mean CV from individual CVs [21].

\section{Results}

Descriptive summaries of the COP-based measure for test and retest sessions and in both groups are presented in Tables 2. ICC and its 95\% CI, SEM, MMDC and CV have been summarized in Table 3 . Test and retest mean scores for any COP measures in two conditions did not show any significant difference, thus indicating the absence of any systematic bias $(\mathrm{p}>0.05)$. The results of reliability analysis were different for COP measure across two test conditions. Also there was not seen uniform observational pattern in each group.

\begin{tabular}{|c|c|c|c|c|c|}
\hline \multirow[b]{2}{*}{ Group } & \multirow[b]{2}{*}{ Variable } & \multicolumn{2}{|c|}{ High Speed } & \multicolumn{2}{|c|}{ Low Speed } \\
\hline & & Test mean (SD) & $\begin{array}{l}\text { Retest mean } \\
\text { (SD) }\end{array}$ & $\begin{array}{l}\text { Test mean } \\
\text { (SD) }\end{array}$ & Retest mean(SD) \\
\hline \multirow{8}{*}{$\begin{array}{l}\text { Healthy Subjects } \\
\qquad(\mathrm{n}=12)\end{array}$} & \multicolumn{5}{|c|}{ AP } \\
\hline & SD of amplitude & $2.58(0.73)$ & $2.74(0.91)$ & $2.62(0.88)$ & $2.70(0.95)$ \\
\hline & SD of velocity & $16.7(4.88)$ & $16.1(4.54)$ & $10.30(2.97)$ & $10.4(3.91)$ \\
\hline & \multicolumn{5}{|c|}{ ML } \\
\hline & SD of amplitude & $1.23(0.26)$ & $1.17(0.24)$ & $0.98(0.28)$ & $1.03(0.26)$ \\
\hline & SD of velocity & $9.06(2.26)$ & $8.14(1.41)$ & $51.3(1.01)$ & $5.14(0.90)$ \\
\hline & Mean total velocity & $1.63(0.47)$ & $1.71(0.56)$ & $1.67(0.58)$ & $1.58(0.47)$ \\
\hline & Path length & $14.7(4.41)$ & $14.1(4.14)$ & $8.93(2.64)$ & $9.14(3.06)$ \\
\hline \multirow{8}{*}{$\begin{array}{c}\text { Low back pain } \\
\text { subjects }(n=12)\end{array}$} & \multicolumn{5}{|c|}{ AP } \\
\hline & SD of amplitude & $2.89(0.64)$ & $2.96(0.48)$ & $2.73(0.82)$ & $3.07(0.66)$ \\
\hline & SD of velocity & $16.5(5.66)$ & $16.3(2.02)$ & $10(2.01)$ & $10.8(2.09)$ \\
\hline & \multicolumn{5}{|c|}{ ML } \\
\hline & SD of amplitude & $1.32(0.43)$ & $1.18(0.25)$ & $1.03(0.22)$ & $0.93(0.16)$ \\
\hline & SD of velocity & $8.32(2.88)$ & $8.11(1.55)$ & $5.02(1.22)$ & $4.70(0.85)$ \\
\hline & Mean total velocity & $1.71(0.53)$ & $1.59(0.43)$ & $1.64(0.51)$ & $1.74(0.52)$ \\
\hline & Path length & $13.7(4.65)$ & $13.5(2.15)$ & $8.76(1.45)$ & $9.3(1.97)$ \\
\hline
\end{tabular}

COP: center of pressure; S.D.: standard deviation; AP: anteroposterior; ML: mediolateral.

Units of COP measures are as follows: cm (SD of amplitude and path length); $\mathrm{cm} / \mathrm{s}$ (SD of velocity and mean total velocity).

Table 2: Descriptive data for COP measures in two conditions of dynamic trunk movement. 


\section{Ergonomics International Journal}

\begin{tabular}{|c|c|c|c|c|c|c|c|c|c|}
\hline \multirow[b]{2}{*}{ Group } & \multirow[b]{2}{*}{ Variable } & \multicolumn{4}{|c|}{ High Speed } & \multicolumn{4}{|c|}{ Low Speed } \\
\hline & & $\begin{array}{c}\text { ICC } \\
(95 \% \mathrm{CI})\end{array}$ & SEM & MMDC & CV & $\begin{array}{c}\text { ICC } \\
(95 \% \mathrm{CI})\end{array}$ & SEM & MMDC & CV \\
\hline \multirow{8}{*}{$\begin{array}{l}\text { Healthy } \\
\text { subjects } \\
(n=12)\end{array}$} & \multicolumn{9}{|c|}{ AP } \\
\hline & SD of amplitude & $\begin{array}{c}\mathbf{0 . 8 2} \\
(0.50 .94)\end{array}$ & 0.34 & 0.67 & $4.66 \%$ & $\begin{array}{l}\mathbf{0 . 8 9} \\
(0.67 \\
0.96) \\
\end{array}$ & 0.3 & 0.58 & $9.27 \%$ \\
\hline & SD of velocity & $\begin{array}{l}\mathbf{0 . 9 2} \\
(0.77 \\
0.97) \\
\end{array}$ & 1.26 & 2.48 & $9.07 \%$ & $\begin{array}{l}\mathbf{0 . 9 7} \\
(0.91 \\
0.99) \\
\end{array}$ & 0.51 & 1.01 & $4.25 \%$ \\
\hline & \multicolumn{9}{|c|}{ ML } \\
\hline & SD of amplitude & $\begin{array}{c}\mathbf{0 . 6} \\
(0.09 \\
0.86) \\
\end{array}$ & 0.14 & 0.27 & $9.77 \%$ & $\begin{array}{c}\mathbf{0 . 6 5} \\
(0.320 .83)\end{array}$ & 0.17 & 0.33 & $9.01 \%$ \\
\hline & SD of velocity & $\begin{array}{c}\mathbf{0 . 6 5} \\
(0.380 .74)\end{array}$ & 1.48 & 2.92 & $10.98 \%$ & $\begin{array}{l}\mathbf{0 . 8 2} \\
(0.49 \\
0.94) \\
\end{array}$ & 0.41 & 0.8 & 2.39 \\
\hline & $\begin{array}{l}\text { Mean total } \\
\text { velocity }\end{array}$ & $\begin{array}{l}\mathbf{0 . 7 6} \\
(0.37 \\
0.92) \\
\end{array}$ & 0.24 & 0.48 & $5.74 \%$ & $\begin{array}{c}\mathbf{0 . 8 4} \\
(0.56 \\
0.95)\end{array}$ & 0.2 & 0.39 & $9.81 \%$ \\
\hline & Path length & \begin{tabular}{|l|}
$\mathbf{0 . 9 3}$ \\
$(0.79$ \\
$0.98)$ \\
\end{tabular} & 1.03 & 2.02 & $13.16 \%$ & $\begin{array}{c}\mathbf{0 . 9 3} \\
(0.80 .98)\end{array}$ & 0.72 & 1.42 & $8.54 \%$ \\
\hline \multirow{8}{*}{$\begin{array}{l}\text { Low back pain } \\
\text { subjects } \\
(n=12)\end{array}$} & AP & & & & & & & & \\
\hline & SD of amplitude & $\begin{array}{l}\mathbf{0 . 6 1} \\
(0.07 \\
0.87) \\
\end{array}$ & 0.36 & 0.7 & $4.93 \%$ & $\begin{array}{c}\mathbf{0 . 6 2} \\
(0.14 \\
0.87) \\
\end{array}$ & 0.42 & 0.83 & $10.89 \%$ \\
\hline & SD of velocity & $\begin{array}{c}\mathbf{0 . 2 5} \\
(0.40 .71)\end{array}$ & 3.7 & 7.2 & $19.6 \%$ & $\begin{array}{c}\mathbf{0 . 4 6} \\
(0.070 .8)\end{array}$ & 1.48 & 2.91 & $4.13 \%$ \\
\hline & \multicolumn{9}{|c|}{ ML } \\
\hline & SD of amplitude & $\begin{array}{r}\mathbf{0 . 1 6} \\
(0.41 \\
0.65) \\
\end{array}$ & 0.31 & 0.61 & $25.4 \%$ & $\begin{array}{l}\mathbf{0 . 1 3} \\
(0.39 \\
0.62) \\
\end{array}$ & 0.17 & 0.33 & $13.96 \%$ \\
\hline & SD of velocity & $\begin{array}{l}\mathbf{0 . 2 6} \\
(0.39 \\
0.71) \\
\end{array}$ & 2.01 & 3.9 & $15.3 \%$ & $\begin{array}{l}\mathbf{0 . 6 9} \\
(0.25 \\
0.89)\end{array}$ & 0.56 & 1.1 & $18.15 \%$ \\
\hline & $\begin{array}{l}\text { Mean total } \\
\text { velocity }\end{array}$ & $\begin{array}{c}\mathbf{0 . 7 4} \\
(0.34 \\
0.91) \\
\end{array}$ & 0.22 & 0.43 & $7.06 \%$ & $\begin{array}{l}\mathbf{0 . 6 7} \\
(0.18 \\
0.89) \\
\end{array}$ & 0.3 & 0.58 & $7.09 \%$ \\
\hline & Path length & $\begin{array}{l}\mathbf{0 . 3 4} \\
(0.31 \\
0.75) \\
\end{array}$ & 2.98 & 5.85 & $16.43 \%$ & $\begin{array}{l}\mathbf{0 . 3 9} \\
(0.18 \\
0.77)\end{array}$ & 1.34 & 2.64 & $4.44 \%$ \\
\hline
\end{tabular}

Table 3: Reliability analysis of COP measures in different two conditions. (Healthy and LBP subjects)

In healthy subjects, all of the parameters in two conditions showed good to excellent reliability with ICCs between 0.60-0.97. Among them, the SDx and SDv parameters showed the highest and lowest value, respectively (Table 3). Results were different in LBP subjects compared to healthy group. Our results demonstrated an acceptable reliability for $\mathrm{Vm}$ and SDx parameters. Also SDVy in high speed condition showed good reliability (ICC=0.69). Rest of the parameters didn't show acceptable value. It seems that the $\mathrm{Vm}$ variable was the common reliable measure in two conditions and between two groups. Consistent with relative reliability, absolute reliability was higher for Vm parameter. For all COP-based measures, higher ICCs were obtained in healthy group. 


\section{Ergonomics International Journal}

\section{Discussion}

To the best of our knowledge, little studies were conducted on the intersession reliability of a set of COP summary measures in healthy and low back pain subjects in different dynamic trunk movement conditions. The COP based parameters showed different levels of reliability in each groups. But Vm and SDx are the most reliable parameter with good to excellent reliability in both groups. In healthy group, these two parameters had higher value in compared to LBP subjects. This result is accordance with previous reports for static task conditions. Salavati, et al. [23] reported excellent ICC value for COP velocity in a group with musculoskeletal disorders. In another study, Lin, et al. [24] also showed excellent reliability for $\mathrm{Vm}$ in younger and older subjects. However due to different measurement protocol (i.e., different sampling duration, test condition, age, analysis methods) it may not be possible to directly compare our results with those published elsewhere.

In LBP patients as the same as healthy subjects, SDx and $\mathrm{Vm}$ showed good reliability but in the rest of parameters is not found high reliability. Although LBP subjects were assessed in relatively pain free period, it seems that the residual motor control changes have effects on movement pattern [21] and consequently on the COP parameters. In healthy subjects more parameters showed acceptable reliability. Decreased reliability for the LBP group was expected, since postural control of LBP subjects is compromised $[25,26]$ and probably they have had problem in controlling body alignment. One of the most important reasons for such results addresses back to task nature. Reliability assessment studies had focused on quiet standing posture, but our trunk bending task required more controlled movement of COG through whole body dynamic motion.

The trunk bending tasks requires the COG be controlled within and out of base of support. Also the change in the COP may be related to joint moments needed to maintain body alignment to prevent of falling [27]. Therefore, it is may be the LBP subjects completed the task more variable than healthy subjects especially in high speed condition and render the COP in a less stable pattern. Hence we saw less reliability in the COP driven parameters for LBP patients. The pattern found for absolute reliability indices was similar to what mentioned for the relative reliability. Absolute reliability was higher for $\mathrm{Vm}$ in most of the test conditions, indicating small measurement errors across repeated measurements. The estimated MMDC for each COP parameter represents the lower bound of the clinically significant change that can be expected when $\mathrm{COP}$ is used as an outcome variable in clinical trial. Future studies must consider the effect of gender on reliability of dynamic trunk movement's postural stability.

\section{Conclusion}

In conclusion, COP measurement demonstrated better test-retest reliability in healthy subjects than LBP subjects. In both groups mean total velocity, as static test condition in previous researches, showed high reliability. This suggests that mean velocity can be an appropriate parameter in evaluation postural control in dynamic task conditions. Further researches are needed to determine the sensitivity of this parameter to detect changes due to clinical interventions.

\section{Acknowledgment}

The authors are grateful to the Terabit Modares University, Tehran, Iran, for the financial supports and the department of ergonomics at University of Social Welfare and Rehabilitation Sciences, Tehran, Iran, for providing lab facilities.

\section{Conflict of Interest}

The authors declare, there were no conflicts of interest affiliations associated with publication of this manuscript.

\section{References}

1. Geldhof E, Cardon G, De Bi, Danneels L, Coorevits P, et al. (2006) static and dynamic standing balance: testretest reliability and reference values in 9 to 10 year old children. Eur J pediatr 165(11): 779-786.

2. Claeys K, Brumagne S, Dankaerts W, Kiers H, Janssens L (2011) Decreased variability in postural control strategies in young people with non-specific low back pain is associated with altered proprioceptive reweighting. Eur J Appl Physiol 111(1): 115-123.

3. Sarti MA, Lison JF, Monfort M, Fuster MA (2001) Response of The flexion-relaxation phenomenon relative to the lumbar motion to load and speed. Spine (Phila Pa 1976) 26(18): E421-E426.

4. Visser JE, Carpenter MG, Van Der KH, Bloem BR (2008) The clinical utility of posturography. Clin Neurophysiol 119(11): 2424-2436.

5. Didomenico A, Gielo-Perczak K, Mcgorry RW, Chang CC (2010) Effects of simulated occupational task parameters on balance. Applergon 41(3): 484-489. 


\section{Ergonomics International Journal}

6. Granata KP, England SA (2006) Stability of dynamic trunk movement. Spine (phila pa 1976) 31(10): E271-E276.

7. Sparto PJ, Parnianpour M, Reinsel TE, Simon S (1997) The effect of fatigue on multijoint kinematics and load sharing during a repetitive lifting test. Spine (phila pa 1976) 22(22): 2647-2654.

8. Shin G, Nance ML, Mirka GA (2006) Differences in trunk kinematics and ground reaction forces between older and younger adults during lifting. Int J Industrial Ergonomics 36(9): 767-772.

9. Bazrgari B, Nussbaum MA, Madigan ML (2012) Estimation of trunk mechanical properties using system identification: effects of experimental setup and modelling assumptions. Comput Methods Biomech Biomed Engin 15(9): 1001-1009.

10. Kollmitzer J, Oddsson L, Ebenbichler G, Giphart J, Deluca CJ (2002) Postural control during lifting. J Biomechanics 35(5): 585-594.

11. Asgari M, Sanjari MA, Mokhtarinia HR, Sedeh SM, Khalaf K, et al. (2015) The Effects of movement speed on kinematic variability and dynamic stability of the trunk in healthy individuals and low back pain patients. Clin Biomech 30(7): 682-688.

12. Lafond D, Champagne A, Descarreaux M, Dubois JD, Prado JM, et al. (2009) Postural control during prolonged standing in persons with chronic low back pain. Gait Posture 29(3): 421-427.

13. Mokhtarinia HR, Sanjari MA, Chehrehrazi M, Kahrizi S, Parnianpour M (2016) Trunk coordination in healthy and chronic nonspecific low back pain subjects during repetitive flexion-extension tasks: effects of movement asymmetry, velocity and load. Hum Mov Sci 45: 182-192.

14. Price DD, Mcgrath PA, Rafii A, Buckingham B (1983) The validation of visual analogue scales as ratio scale measures for chronic and experimental pain. Pain 17(1): 45-56.

15. Chehrehrazi M, Sanjari MA, Mokhtarinia HR, Jamshidi AA, Maroufi N, et al. (2017) Goal equivalent manifold analysis of task performance in non-specific LBP and healthy subjects suring repetitive trunk movement: effect of load, velocity, symmetry. Hum Mov Sci 51: 72-81.

16. Dupeyron A, Rispens SM, Demattei C, Van Dieën JH (2013) Precision of estimates of local stability of repetitive trunk movements. Eur Spine J 22(12): 2678-85.

17. Denegar CR, Ball DW (1993) Assessing reliability and precision of measurement: an introduction to intraclass correlation and standard error of measurement. J Sport Rehabilitation 2(1): 35-42.

18. Shrout PE, Fleiss JL (1979) Intraclass Correlations: Uses in assessing rater reliability. psychol bull 86(2): 420-428

19. Atkinson G, Nevill AM (1998) Statistical methods for assessing measurement error (reliability) in variables relevant to sports medicine. Sports Med 26(4): 217238.

20. Cicchetti DV, Sparrow SA (1981) Developing criteria for establishing interrater reliability of specific items: applications to assessment of adaptive behavior. Am J Ment Defic 86(2): 127-137.

21. Steffen T, Seney M (2008) Test-retest reliability and minimal detectable change on balance and ambulation tests, the 36-item short-form health survey, and the unified parkinson disease rating scale in people with parkinsonism. Phys Ther 88(6): 733746.

22. Shafeei A, Mokhtarinia HR, Maleki-Ghahfarokhi A, Piri L (2017) Cross-cultural adaptation, validity, and reliability of the persian version of the orebro musculoskeletal pain screening questionnaire. Asian Spine J 11(4): 520-530.

23. Salavati M, Hadian MR, Mazaheri M, Negahban H, Ebrahimi I, et al. (2009) Test-retest reliabty of center of pressure measures of postural stability during quiet standing in a group with musculoskeletal disorders consisting of low back pain, anterior cruciate ligament injury and functional ankle instability. Gait Posture 29(3): 460-464.

24. Lin D, Seol H, Nussbaum MA, Madigan ML (2008) Teliability of cop-based postural sway measures and age-related differences. Gait Posture 28(2): 337-342.

25. Mientjes MI, Frank JS (1999) Balance in chronic low back pain patients compared to healthy people under various conditions in upright standing. Clin Biomech(bristol, avon) 14(10): 710-716.

26. Nies N, Sinnott PL (1991) Variations in balance and body sway in middle-aged adults. Subjects with healthy backs compared with subjects with low-back dysfunction. Spine (phila pa 1976) 16(3): 325-330. 
27. Geurts AC, Nienhuis B, Nulder TW (1993) Intrasubject variability of selected force-platform parameters in the quantification of postural control. Arch Phy Med Rehabilitation 74(11): 1144-1150. 